\title{
MAS EVIDENCIAS SOBRE EL DESARROLLO DE LA CULTURA SELVA TROPICAL EN LA COSTA NORTE DE COLOMBIA, DURANTE EL PRIMERO Y SEGUNDO MILENIO ANTES DE CRISTO
}

\author{
Donal W. Foster y Donal W. Lathrap \\ Traducido por: \\ Juan A. Yangüiez \\ De la Revista \\ Journal of the steward Antropological Society \\ Vol. 4, No. 2 Spring, 1973. pp. 160-199 Urbana, \\ Illinois.
}

El material presentado en este informe es el resultado de una continua investigación en biblioteca urchive durante 19(88-1972. Agradecemos a Robert E. Jarboe por su estímulo y crítica en este informe. tambien queremos agradecer la ayuda y cooperación de Aquiles Escalante. Director del Museo Arqueológi11i. Barranquilla, Colombia, S. A. 


\section{INTRODUCCION}

La continuación de las investigaciones en la prehistoria Suramericana, sugiere que las tierras bajas tropicales fueron un centro mayor para los movimientos en gran escala de la población, evolución agrícola y desarrollo cerámico (Lathrap, 1966, 1970; Sauer, 1952, y Cruxent y Rouse 1958-59). Algunos científicos han minimizado la importancia de la Cultura Selva Tropical ${ }^{2}$ en el desarrollo de la civilización Suramericana (Ford 1969; Meggers 1954; Meggers y Evans 1957). Sin embargo se está haciendo más evidente que la difusión de ideas y el movimiento de gente dentro del continente tuvo lugar a lo largo de rutas fluviales, con la cuenca del Amazonas, jugando un papel principal en la expansión de la civilización en Sur América (Lathrap 1970).

Es el record cerámico el que provee al arqueólogo con una fuente primaria para entender la historia cultural temprana en las tierras bajas tropicales de Sur América. Los esfuerzos continuos de los Reichel-Dolmatoff en la Arqueología Colombiana ha provisto a los científicos, interesados en la polémica arriba discutida, con una riqueza de información sobre la historia cerámica del nor-oeste de Sur América (véase ReichelDolmatoff, G. y A. 1951, 1956, 1957 y 1961, y Reichel-Dolmatoff, G. $1954,1955,1965$ a y 1965b. ). Desafortunadamente, la utilización de los trabajos de los Reichel-Dolmatoff para aumentar nuestro conocimiento del hombre prehistórico en Sur América ha sido esporádica.

El informe del sitio de Momil (Reichel-Dolmatoff, G. y A. 1956) ejemplifica este punto. Mientras que este documento provee una de las descripciones más completas de una larga secuencia de ocupación en Sur América, su importancia ha sido disminuida por los autores (véase Reichel-Dolmatoff (1965b). Ha sido citado infrecuentemente y en ocasiones mal usado. (véase Ford 1969). Aún más, puesto que no se estableció un fechamiento cronométrico para la secuencia de Momil, las citas que usualmente se hacen se conforman a las sugerencias originales postuladas por los Reichel-Dolmatoff.

2 La cultura de Selva Tropical es definida económicamente como ${ }^{*} \ldots$ una forma de vida mantenida por una agricultura intensiva de tubérculos. Cuando es posible, hay una máxima explotación de los recur. sos alimenticios de los ríos, lagos y costas; mientras que la caza de animales terrestres y aves en las sel. vas lejos de las principales arterias acuáticas fue definitivamente de importancia secundaria (Lathrap $1970:(77)$ 104
El propósito de este informe es el de evaluar la secuencia de Momil en base al actual record arqueológico del norte de Sur América. Se demostrará que las comparaciones con otros sitios locales pueden conducir a una interpretación más clara de este sitio que la que se puede lograr relacionando a Momil con secuencias distantes de Mesoamérica y Perú.

La intrusión de materiales Barrancoides, un complejo de cultura Selva Tropical bien desarrollado de Venezuela, en la cuenca del río Sinú del norte de Colombia, permite un fechamiento más probable para la secuencia de Momil.

\section{DESCRIPCION DE MOMIL}

Las excavaciones en Momil fueron completadas en los principios de 1955 por los Reichel-Dolmatoff. El sitio está localizado en los bordes de una laguna en la cuenca del bajo río Sinú en el norte de Colombia y probablemente fue un poblado ribereño durante su período de ocupación. Momil representa una aldea sedentaria con una larga ocupación pero discontínua situada en una franja plana entre las riberas de la laguna y unos cerros de baja elevación (Reichel-Dolmatoff, G. 1965b:68).

La excavación más grande fue un corte de 2 por 6 metros con una profundidad de 3 metros. Este fue el basurero que dio un total de 336,732 tiestos y cantidades de materiales óseos, líticos y conchas. Debido a la naturaleza exploratoria de la excavación, el único procedimiento factible fue el uso de niveles arbitrarios de $25 \mathrm{cms}$. Sin embargo, la falla de esas unidades artificiales al no corresponder con los niveles naturales, conduce a ciertas dificultades de interpretación. Se da una impresión de mayor continuidad cultural que la que es justificable si se analiza todo el material disponible. (Véase diagrama 1.).

Usando los 25 tipos cerámicos sensibles de Reichel-Dolmatoff (véase Reichel-Dolmatoff, G. y A. 1956: 131 y Tabla 1), se construyó una gráfica de frecuencias describiendo su relativa popularidad. (Véase diagrama 2). Un examen de este gráfico y del perfil de excavación (diagrama 1) sugiere que la estimación de 1000 años hecha por el autor para la secuencia de Momil puede ser muy conservadora. El perfil del suelo indica que hubo 5 sub-períodos en Momil I en vez de los 4 propuestos en el informe. Esto parece estar apoyado por la distribución cerámica. Algunas categorías cerámicas son muy amplias y enmascaran la naturaleza distintiva de las unidades culturales.

La crema Bañada (a) e Incisa Irregular (b) parecen ser exclusivas de los estragos más bajos del nivel 13 y son características de un complejo cerámico separado el cual será designado como Momil IA-1. La Aplicada Simple (c), Acanalada Punteada (d), Acanalada Aplicada A (e) y Acanalada Aplicada B (f) parecen estar mal representadas en la gráfica de frecuencia debido a su proveniencia, parte superior del nivel 13 y parte inferior del 12. Esos cuatro tipos cerámicos parecen marcar una unidad cultural separada la cual se designará Momil IA-2. 
Los niveles 11 y 10 probablemente representan una muestra pura de un componente, ya que los niveles naturales y culturales se alinean perfectamente. Negra Incisa (g), Incisa Fina (h), Punteada (i), y Negativa A (1) son los tipos decorados dominantes en el complejo designado IB por los autores. Los niveles 9 y 8 , a pesar de no estar tan claros como los sub-períodos anteriores, parecen alinearse con Momil IC, y los tipos cerámicos Rojo Bañada (j) y Rojo sobre Blanco (k) se hacen populares aquí. Estos dos tipos cerámicos parecen cubrir varias secuencias ocupacionales así como la Negra Incisa (g) e Incisa Fina (h). El carácter bimodal del Rojo sobre blanco (k) puede deberse a que dos tradiciones cerámicas separadas han sido agrupadas como un solo tipo decorativo y puede que no cubran los períodos Momil I y II como lo sugiere la gráfica de seriación.

La deposición en Momil II está relacionada con una ocupación más continua así como con una continuidad cultural durante este período. Los picos de popularidad de los tipos cerámicos no coinciden temporalmente y no hay una separación clara en ninguno de los 6 niveles superiores. Tres tipos pintados $(\mathrm{m}, \mathrm{n}, \mathrm{o})$ son probablemente exclusivos de Momil II y partes de las colecciones encontradas debajo del nivel 6 son probablemente intrusos en Momil I. Fuera de la Rojo Bañada (j) y de la naturaleza dudosa del Rojo sobre Blanco (k), el gráfico de seriación cerámica sugiere una discontinuidad bien marcada entre Momil I y II. Hay una deposición horizontal de cerámica separando los dos períodos lo cual indica claramente el resultado de la erosión y una subsiguiente redeposición de tiestos en una alta concentración.

Basado en trabajos en ambientes ribereños similares (Lathrap 1968), los depósitos de basureros en medio ambientes de selva tropical no exceden más de $30 \mathrm{cms}$. cada 100 años. Por lo consiguiente, las ocupaciones en Momil solamente pueden representar un período de cerca de 900 años. Además, los hiatos entre cada uno de los 5 sub-períodos de Momil I y la definitiva separación entre Momil ID y Momil II, probablemente están relacionados con los cambios del curso del río Sinú. Permitiendo unos 200 años conservativos para cada uno de los 5 cambios, tendríamos 1000 años adicionales. El resultado conservador es de unos, tendriamos mo mínimo para la secuencia total. Si esta interpretación es correcta, tal espacio temporal podría tener implicaciones significativas para las in terpretaciones de la arqueología Colombiana y para la reconstrucción la historia cultural de Sur América como un todo. Tales implicaciones forman la base de este informe y serán discutidas a continuación.

La secuencia de agricultura de tubérculo seguida por la agricultura de semilla fue evidente en los dos períodos en Momil. En las conclusiones del reporte de Momil, los Reichel-Dolmatoff sugieren que los gran des platos planos circulares con bordes levantados [budare] y la industria de micro-hojas manifestada por la abundancia de piezas de sílex manufacturado, los cuales son encontrados exclusivamente en Momil I, están relacionados con el patrón de subsistencia basado en la yuca brava mientras que la repentina aparición de manos y metates en Momil II sugieren una economía basada en el maíz (1956:270-272). (Véase diagrama 3).
Una secuencia similar fue reportada por Rouse y Cruxent (1963) en Rancho Peludo, un sitio ribereño en el noroeste de Venezuela cerca de la frontera con Colombia. Aquí las manos y metates aumentan en número a través del tiempo mientras que la incidencia de los comales declina marcadamente, el cambio ocurre a mediados del primer milenio antes de Cristo. Se ha sugerido que el estilo Rancho Peludo, representado por un sistema agrícola de tubérculos de manioca, fue reemplazado por un sistema de cultivo de semillas de maíz, caracterizado por el estilo Guasare $(1963: 54,56)$. Más evidencias sobre una temprana introducción de agricultura de maíz se han encontrado en los ríos Cesar y Ranchería, distrito intermedio entre los ríos Magdalena y Sinú en el noroeste de Colombia y el distrito del Lago Maracaibo en el este de Venezuela (Reichel-Dolmatoff, G. y A. 1951:288 y Reichel-Dolmatoff, G. 1965b:117).

Puesto que no se ha hecho un fechamiento absoluto para los niveles de Momil, la estimación de esta secuencia debe depender en las relaciones con otros sitios conocidos y desarrollados que han tenido lugar en y alrededor del norte de Colombia durante el primer y segundo milenio antes de $\mathrm{Cristo}^{3}$. Las comparaciones que se presentarán estarán basadas fuertemente en las técnicas estilísticas y decorativas de los materiales cerámicos. Como consecuencia, los argumentos presentados reforzarán las evidencias cualitativamente en vez de cuantitativamente.

\section{DESCRIPCION DE CIENAGA DE ORO}

El sitio tipo de Ciénaga de Oro está situado en una zona ecológica idéntica a la de Momil pero localizado río arriba en el medio río Sinú (Véase mapa, diagrama 4, y Reichel-Dolmatoff G. y A. 1957:85-102). El sitio representa una aldea sedentaria con una duración aproximada de 400 años. Basado en la similitud de su medio ambiente con el de Momil, uno puede esperar que Ciénaga de Oro tenga una adaptación comparable.

La excavación se hizo en el barrio Panagua, Ciénaga de Oro. Se excavó un pozo de sondeo de 2 por tres metros hasta una profundidad de un poco menos de cuatro pies. Al igual que en Momil, se usaron niveles arbitrarios de $25 \mathrm{cms}$. para separar el material. Sin embargo, la estratigrafía de Ciénaga de Oro no estaba tan bien definida como en Momil y las tradiciones culturales no eran obvias, estaban ausentes. La mayoría del material excavado de Ciénaga de Oro sugiere una ocupación continua. Los autores (1957:87-94) describieron cinco tipos decorativos de cerámica Mientras que no era evidente un hiato estratigráfico, la aparición repen-

3 En la literatura se han mencionado posibles afinidades más allá del área geográfica inmediata. Se 3 En la literatura se han mencionado posibles afinidades más allá del área geográfica inmediata.
ún Lathrap, "El cuñeado verdadero es uno de los pocos rasgos discutidos por (Alicia y Gerardo Rechel-Dolmatoff) que puede tener un valor específico como un marcador de horizonte. Ocurre en Tlatilco La Venta alrededor de $1000-800$ a. de Cristo y entre los complejos del norte del Perú alrededor de 800 . 600. Es tentedor asta base, todo Momil I debe pre-da rar los 800 a $C$ y su parte más preprana debe comenzar en el segundo milenio ya que el pre-datar los 800 a. de C. y su parte más temprana debe comenzar en el segundo milenio ya que el cuñeado
verdadero ocurre solo en los niveles 5 y $6(1958: 361-362)^{*}$. El cuñeado verdadero aparece solamente en Momil en el nivel 6. (Véanse lámina 6-a, diagrama 2 (cuñeado) y Reichel-Dolmatoff, G. y A. 1956:166 168 y lámina XV 1-4,7). 
tina de dos tipos cerámicos, Incisa Ancha y Modelada (véase diagrama 5 ), puede relacionarse con una reocupación posterior del sitio. Puesto que los materiales intrusivos se encuentran en el nivel 1 , hay un problema en su interpretación debido a los métodos de excavación. Dada la naturaleza del material, hay dos explicaciones posibles. Esos dos tipos de cerámica representan vasijas de prestigio, raras, adquiridas por comercio, una capa de ocupación de 5-10 cms. cubriendo un depósito ocupaciona de Ciénaga de Oro puro. Esta última interpretación es más favorable y puede comprobarse con futuras excavaciones.

Como resultado de sus excavaciones, los Reichel-Dolmatoff concluye ron que Ciénaga de Oro se derivó de Momil II. Esto, ellos arguyen, se debe a la preponderancia de rasgos que Ciénaga de Oro tiene en común con Momil II, y por la ausencia de rasgos significantes claramente identificados con Momil I. Por ejemplo, los sellos tubulares cilíndricos, silbato aviformes y adornos biomorfos aparecen solamente en Momil II y están claramente presentes en Ciénaga de Oro, mientras que la industria sílex de Momil I está ausente en Ciénaga de Oro (1957:127-128).

Hay evidencias en ambos sitios que sugieren que tal interpretación puede no ser correcta. Hay un número importante de rasgos característicos de Momil II que no están presentes en el complejo Ciénaga de Oro. Aún más, existe un número de rasgos comunes en ambos, Momil I y Ciénaga de Oro los cuales están ausentes en Momil II. En el análisis que sigue, se presenta un modelo para relacionar ambos sitios.

\section{COMPARACIONES MOMIL-CIENEGA DE ORO}

Además de la industria sílex, otros rasgos característicos de Momil I están ausentes en Ciénaga de Oro: los budares, decoración con un baño crema y sellos planos para decorar. Cono lo postularon los Reichel-Dolmatoff, es muy dudoso que Ciénaga de Oro se haya derivado directamente de Momil I. Aún más, sería muy difícil seriar el material de Ciénaga de Oro entre cualquiera de las fases de Momil I en base a las figurinas. Las posibilidades son $^{1}$ colocar Ciénaga de Oro después de Momil II tal como lo sugieren los Reichel-Dolmatoff; ${ }^{2}$ seriarlo entre algunos de los niveles de Momil II (lo cual es una posibilidad débil debido a la naturaleza homogénea de este período), ó (3) colocarlo entre Momil I y Momil II o muy tempranamente en Momil II, digamos niveles 5 y 6 . Esta última posibilidad ha sido sugerida por Henning Bishof (comunicación personal) como lo más probable.

Las figurinas constituyen el mejor artículo de análisis. En Momil se puede hacer una distinción muy clara entre las figurinas encontradas en el nivel I y el nivel II. Las de los niveles más bajos son pesadas y sólidas, muy estilizadas y la mayoría están en posición sentada. Los brazos tienen forma de manijas curvas en cada lado y los rasgos corporales están limitados a perforaciones en la cabeza para indicar la boca y orejas y en el estómago para representar el ombligo (véase Figura 1 y Reichel-Dolmatoff, G. 1965b: lámina 5). Por otra parte, las figurinas de Momil II son 108 bastante delicadas y generalmente huecas. Esto explica por qué no se encuentran figurinas completas. Las figurinas de Momil II son considerablemente mas realistas en su forma, tienen mejores proporciones y detalles corporales que las encontradas en Momil I. (Véase Figura 2.). Las extremidades están ejecutadas con gran cuidado y las expresiones faciales ton bien detalladas, especialmente los ojos, nariz, orejas y boca. Con freson bien detalladas, especialmente los ojos, nariz, orejas y boca. Con frecuencia se indican adornos personales tales como brazaletes y collares y la nariz es típicamente de base levantada. Se han encontrado figurinas tanto paradas como sentadas.

La manufactura de figurinas en Ciénaga de Oro sugiere un cuadro de (Reichel-Dolmatoff, G. y A. 1957: 97-98).

\section{Ciénaga de Oro}

\begin{tabular}{ccr} 
Nivel & \multicolumn{2}{r}{ Tipos de Figurina } \\
& A & B \\
1 & 1 & 2 \\
2 & 4 & 6 \\
3 & - & 3 \\
4 & - & 6 \\
5 & - & -
\end{tabular}

(adaptado de Reichel-Dolmatoff, G. A. 1957: 97)

El tipo A aparece solamente en los dos niveles superiores mientras el tipo $\mathrm{B}$ aparece claramente desde el nivel 1 hasta el 4 . El tipo A

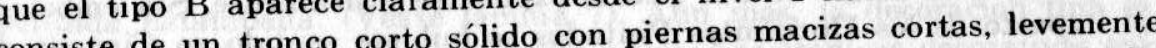
consiste de un tronco corto solido con pienta cóncava. Los brazos consiscurvas y que terminan en un pienco La ten en cortas protuberancias cónicas que salen lateralmente del tronco. La cabeza es generalmente hueca y la nariz es ancha y de base levantada. Los rasgos faciales están generalmente representados por líneas ancha Los rasgos faciales estan senos, incisas. Dos pequeñas protuberancias modeladas réprese láminas 8-b, d, g y Reichel-Dolmatoff, G. 1965b: lámina 6).

El tipo B carece de decoración incisa, los rasgos faciales están crudamente modelados y presentan pocos detalles. (Véanse láminas 8-a, e y Reimente los rasgos decochel-Dolmatoff, G. y A. 1957: lamina XIII-1). Mientras lósica es muy sirativos en el tipo de figurina $\mathrm{B}$ son mínimos su forma basica es muy similar al tipo A. (Compárese la lámina 8-a con la lámina 8-b). El análimilar al tipo A. Ciénaga de Oro.

La combinación de partes sólida y hueca en una misma figurina, la con detalles faciales, nariz con presencia de figurinas con representacionas con reproducciones realistas base levantada, y las piernas bien formadas con reprosico del Tipo A) junto con los modelos de las extremidades (característico del Tipo A) junto con los modelo. que no son completamente descriptivos (Tipo B) sugieren que las figuri 
nas de Ciénaga de Oro poseen un número de diseños característicos de los dos períodos representados en Momil. Sin embargo, no hay un com plejo de rasgos paralelos entre Ciénaga de Oro y Momil en la manufactu ra de figurinas.

Como se mencionó arriba, la seriación de las figurinas de Ciénaga de Oro entre cualquiera de los niveles de Momil I sería compleja. Es difícil imaginarse que los artesanos, cuya cultura adquirió una tecnología la cual permitió la manufactura de formas altamente humanizadas halladar en Momil II, se revirtieran a la manufactura de formas sin vida que se encuentran en la mayoría de las figurinas de Ciénaga de Oro (Tipo B) y poseyesen dichas figurinas por comercio. En esta base, es difícil ver co mo Ciénaga de Oro puede seguir a Momil II. Una explicación más pro bable sería que las figurinas de Ciénaga de Oro están relacionadas con una tradición que evolucionó de las figurinas de Momil I. Se han sugeri do paralelos entre las características de la forma pesada del cuerpo y técnicas decorativas crudas utilizadas en los rasgos del cuerpo

En la descripción de los materiales de Momil, los Reichel-Dolmatoff dicen que la tradición de decoración pintada bícroma se había estableci. do antes del final de Momil I (1956:152-154). Este estilo pintado paralelo al material Panagua Rojo sobre Ocre (véase diagrama 5) se encuentri desde los niveles más bajos en Ciénaga de Oro y aparece en Momil du. rante la fase IB. Lo más significativo, sin embargo, es la ausencia de vasijas polícromas y otros ejemplares bícromos en Ciénaga de Oro. Si Ciéna. ga de Oro se derivase de Momil II, sería difícil ver como tal derivación pudo ser tan selectiva. Se puede argüir que las vasijas polícroma y bí. croma en Momil comprenden una colección de vasijas comerciadas y que puesto que sus miembros disminuyeron en los niveles finales en Momil, su ausencia en Ciénaga de Oro podría explicarse lógicamente. Sin embargo. el impacto de los Horizontes Pintados se hubiera sentido en Ciénaga de Oro, si este complejo [Ciénaga de Oro] hubiera seguido a Momil II, como lo sugirieron los Reichel-Dolmatoff (véase Reichel-Dolmatoff, G. 1965b:45). Parece más razonable postular que la cerámica polícroma de la secuencia La Loma se derivó en parte, de Momil II y no son objeto de comercio en Momil (1965b: 120)

En las manifestaciones tempranas de la cultura de Selva Tropical en el río Orinoco en Venezuela (la Tradición Saladoide), el engobe rojo aparece como una técnica decorativa diagnóstica. La pintura roja sobre superficie sin pintar y blanco sobre rojo son comunes (Cruxent y Rouse 1958-9:213-223). Esta alfarería parece ser paralela con la presencia temprana del engobe rojo en $\mathrm{Momil}^{4}$ y Ciénaga de Oro

Momil Dentada Zonificada, mientras que se encontró en todos los niveles de la secuencia de Momil, su pico de popularidad ocurre en el $\mathrm{ni}$ vel 7, 1.e en las partes finales de Momil I (véanse láminas 5-a-f, 1 y 6-b-i y Reichel-Dolmatoff, G. y A. 1956: láminas VII 4-10, IX 6-9 y XI 1-9).

4 Aqui se hace referencia a Momil Rojo sobre Blanco. Otros tres escilos pintados han sido defiui. dos por los autores: Policromada. Negro sobre Blanco y Negro sobre Rojo. Ellos parecen ser intruses en los

thete tipo cerámico tiene paralelos muy cercanos con Panagua Dentada liutampada en Ciénaga de Oro. (V́éanse lámina 7 y Reichel-Dolmatoff, G v A. 1957: lámina XI 1-6). Los motivos de diseño dentado son populares linto en Ciénaga de Oro como en Momil (1957:127). En Momil esta tratision se encuentra con incisiones de línea fina y ancha (Reichel-Dolmatulf, G, y A. 1956: lámina XVI 3,4. (Incisa Curvilinear) y lámina XVI 9. 10 (Dentada Ranurada) y en cerámica pintada (1956: lámina XV 8-10).

Los sellos cilíndricos de Ciénaga de Oro son muy similaies a los enenutrados en Momil II (compárense lámina 3-e,h,i con Reichel-Dolmatwil G. y A 1956: lámina XXIV 1-nos. 3,4) y los silbatos encontradien en Ciénaga de Oro son algo similares a los encontrados en todos los niveles de Momil. (Compárese Reichel-Dolmatoff, G. y A. 1957: lámina KIII 8,18.21-22 con Reichel-Dolmatoff G. y A. 1956: figura 11-nos. 17-19).

Momil II representa la llegada de un nuevo patrón de subsistencia in el río Sinú. En este período, la agricultura de semilla: maíz, parece feemplazar la larga secuencia de agricultura de tubérculos de manioca. Lin manos y metates aparecen por primera vez en los inicios de Momil II mientras que la tradición de micro-hojas y los burare se encuentran enelusivamente en los 3 niveles más bajos de Momil I. Como se observa iil el diagrama 6 , los budares y la industria de sílex están ausentes en Cienaga de Oro. Sin embargo hay evidencias de la presencia de piedras to moler en este sitio. (Véase diagrama 6 , nota 10).

La evidencia adicional, para la creación de los materiales de Ciénaun de Oro entre Momil I y Momil II, se encuentra en el área de decoraHîn cerámica. El "rocker-stamping" verdadero aparece solo en los niveles maa tempranos de Momil II (véase el tipo cerámico cuñeado, diagrama 2 V lamina 6-a), y es una técnica popular en Ciénaga de Oro, Panagua Wentada Estampada. (Véase lámina 7). Aún más, la decoración fina es Haraintente en las cerámicas de ambos sitios. Las técnicas decorativas en imitradas en la cerámica Momil Incisa Fina (Reichel-Dolmatoff, G. y A 1456: 147) tiene algunos paralelos con los materiales Ciénaga de Oro Inciä Fina (véase Reichel-Dolmatoff, G. y A. 1957:91). Aún más, las puntas vbjetos tubulares se encuentran en ambas colecciones y hay evidencias ie un desarrollo paralelo del appliqué indentado. (Compárese ReichelWolmatoff, G. y A. 1956: lámina VI 7 y lámina VIII 1,3 con ReichelDolmatoff, G. y A. 1957: lámina XI 19). Finalmente hay similitudes en lan formas de vasijas de Momil I y Ciénaga de Oro: vasijas globulares $60 n$ bordes anchos evertidos, receptáculos hemisféricos con bordes rectos $\checkmark$ vasijas con una quilla fuerte (silueta compuesta) ${ }^{5}$

Se ha construído una gráfica de seriación basada en rasgos específi10i, fácilmente identificables para relacionar las colecciones de Momil (6i) las de Ciénaga de Oro. Este método de seriación está basado en la mresencia o ausencia de rasgos específicos en vez de la frecuencia de cual-

Desafortunadamente, no hay suficientes descripciones de las formas de vasijas, especialmente en el in Cienaga de Oro, para intentar cualquier comparación cuantitativa basada en la forma del cuer. iii. iit tampoco fue posible delimitar la relación entre las formas específicas de los bordes y las formas de 
quier rasgo individual y se puede aplicar a una gama de materiales, incluyendo especímenes raros o no asociados así como materiales encontrados en cualquier clase de asociación arqueológica (Rowe, 1961: 328). Puesto que la información cuantitativa para propósitos comparativos entre Momil y Ciénaga de Oro. (Véanse diagrama 6 y las notas al pie de página adjuntas).

La distribución de esos rasgos tiende a verificar la sugerencia de Bishof, i,e. que Ciénaga de Oro se seriaría mejor entre Momil I y II. Es obvio, sin embargo, que la colección completa de Ciénaga de Oro no puede derivarse directamente de Momil I ni tampoco conduce directamente a Momil II. Hay rasgos importantes exclusivos tanto para Momil I y Momil II, que no están presentes en Ciénaga de Oro. Aún más, hay rasgos exclusivos a Ciénaga de Oro que están ausentes en todos los niveles de Momil (véase Reichel-Dolmatoff, G. y A. 1957: 128). Son esos rasgos, encontrados exclusivamente en Ciénaga de Oro, los que nos permiten establecer el fechamiento de Momil con bases más firmes.

Los bordes anchos decorados con lóbulos o triángulos modelados que se proyectan desde los bordes de las cerámicas de Ciénaga de Oro, están ausentes en Momil. Este tipo de decoración está asociado con $\mathrm{Pa}$ nagua Incisa Ancha y da la impresión de ser una tradición cerámica separada en Ciénaga de Oro. (Véanse láminas 1 y 2). Hay una gran diversidad en los tipos de bordes en los niveles superiores en Ciénaga de Oro, especialmente con la introducción del borde exótico tipo $\mathrm{H}$. (Véanse lamina 2-a-b,c y e, Figura 3-b y Reichel-Dolmatoff, G. y A. 1957:152). Panagua Incisa Ancha está también asociado con la manufactura de boles pandos con patas largas tubulares o base anular, y decoración en el interior, un estilo de producción cerámico no presente en Momil. (Véase 1957: 95 , Figura $5-\mathrm{s}, \mathrm{t}, \mathrm{u})$.

Otras evidencias que sugieren la intrusión de un complejo en Ciénaga de Oro se pueden observar en las técnicas de cocción usadas en la producción cerámica. Panagua Incisa Ancha (Reichel-Dolmatoff, G. y A. 1957: 89, lámina XII 1-6) y Panagua Modelada (1957:92, lámina XI 7, 15 y lámina XII 9), son los dos tipos cerámicos asociados con el complejo intruso, fueron cocidos en atmósfera reducida mientras que los otros tipos cerámicos fueron cocidos en una atmósfera oxidante 6 . (Véase 1957:88-93).

Este complejo intruso identificado en Ciénaga de Oro es definitivamente parte de una rama de la Tradición Barrancoide. Los bordes evertidos en los boles pandos (véase figura 3 y compárense las láminas 1-a- y 2-a con la figura 4-d y Cruxent y Rouse 1958-9: figura 60-nos. 10, 12a), junto con las bases pedestal, boles hemisféricos, rebordes labiales, cabezas zoomórficas adheridas a bordes evertidos, $y$ volantes de huso (véase diagrama 6 , nota 2 ), todo lo cual sugiere que Ciénaga de Oro estuvo influida por una tradición Barrancoide generalizada. (Compárense Cruxent y Rou-

6 Las vasijas Panagua Modelada están caracterizadas por una tira de cerámica bien definida paralela al borde y se encuentra en la pared exterior de la vasija. Es similar a las técnicas decorativas encontra das en los materiales Barrancoide de Venezuela.

112 e 1958-9:85, y lámina 27-nos. 1-7 con Reichel-Dolmatoff, G. y A. 1957: lámina XIII 9,12,13,15,16,21,22). Este complejo intrusivo en Ciénaga de Oro está marcadamente ausente en Momil pero muy relacionado con las tradiciones cerámicas del este de Venezuela?

Cuando la naturaleza de Ciénaga de Oro es examinada, ésta parece representar una evolución continua en la tradición Momil con la parte más temprana ya algo evolucionada de ID y el final no muy idéntico al comil indicaciones sugieren que el hiato entre $\mathrm{Mo}$ mil I y II propuesto. Por lo conmil I y II es mayor que el periodo de 200 años ya propuesto. Por lo consiguiente, los 1900 años para toda la secuencia parece ser sumamente conservador.

\section{MALAMBO}

Hacia el este y norte de Momil, a lo largo de la margen occidental del río Magdalena, yace el sitio de Malambo. Los restos ocupacionales se encuentran dispersos sobre un área ancha. El depósito consiste de un estrato de cerámica con desgrasante de arena, y huesos de animales cuestrato de cerána de biertos por una cósicos depositos historia desde aproximadamente dez, 1962). Las a $70 \mathrm{~d}$. de C. (1962:86) lo cual sugiere que fue contemporáneo 1120 a. de C. a 70 d. denning Bishof (cocon la parte más reciente de la secuencia de Momil. Henning de Malambo
municación personal) duda que cualquiera de los materiales de Malam sea de gran antigüedad.

Malambo yace a lo largo del borde de una serie de lagunas conectadas de tal manera que la comunicación fluvial al río Magdalena, unos 7 das de tal manera que durante el período kilómetros hacia el este, es posible. Es probable que huriendo cambiado de ocupación, el Magdalena corriera cerca del sitio, habiendo cambiado su curso en tiempos históricos. El sitio es un basurero con muy poca evidencia de recolección de conchas. "Los moluscos no fueron utilizados en la alimentación aunque eran abundantes tanto en las playas cercanas como en las lagunas de agua dulce (Reichel-Dolmatoff, G. 1965b:64)"

Hay una abundancia de huesos de reptiles que junto con la presencia de budares encontrados por todo el depósito, sugiere que Malambo fue un asentamiento de larga duración con una economía de subsistencia basada en la agricultura de tubérculo de selva tropical suplementada con basada en sitio indudablemente tuvieron recursos riberenos. Los ocupantes hes oeste. (Ver algún impacto en los sitios costaneros inmediatamente hacia el oeste. (Ver mapa). De acuerdo con Reichel-Dolmatoff (1956b:59), los materiales de Barlovento datan de 1550-1032 a. de C. sugiriendo un traslapo tempora Barlovento datan de conchas, con Malambo. Es probable que las culturas de mosta el primer presentes en la costa al oeste de Malambo, persistieron hasta el prime milenio después de Cristo. Bishof (1966:489) nota paralelos entre los appli-

Un diseño adicional encontrado en Ciénaga de Oro que se puede relacionar con las técnicas cerà micas populares en Venezuela es el borde cuello de botella. (Véase lámina $4 \mathrm{~h}$ y figura $4 \mathrm{a}, \mathrm{b}, \mathrm{c}$.). 
qué modelados bionıorfos y bordes con puntos con los de Malambo reportados por Angulo Valdez (1962).

Malambo tiene algunas relaciones vagas con la tradición Barrancoide de Venezuela. Estas se pueden ver en las proyecciones circulares en las vasijas, la tendencia de decorar las protuberancias con líneas incisas, la aplicación de pelotitas hemisféricas con un punto perforando su centro, la imitación de adornos modelados antropomorfos y biomorfos, el uso de espirales incisos (compárense Cruxent y Rouse, 1958-9: lámina 28 nos. 5-7,9-11, 13-15 con Angulo Valdez 1962: láminas I e, f,II k, III a-d, IV ah, V g-j, VI b-d), soportes para ollas, cerámica pintada, apéndices grotescos, bases perforadas y budare (Angulo Valdez 1962:83). La técnica decorativa de hileras de puntos sobre una protuberancia de arcilla en la pared de la vasija sugiere paralelos con los materiales Venezolanos. (Compárese Angulo Valdez 1962: lámina III j y VI k con Cruxent y Rouse 1958-9: lámina 30-nos. 5,20). Esta técnica también pudo haber sido usada en Momil. (Véase Reichel-Dolmatoff, G. y A. 1957: lámina XI 9). Sin embargo, puesto que no se han encontrado rebordes labiales en Malambo, es muy dudoso que se puedan establecer afinidades Barrancoides directas. Malambo sí tiene algunos paralelos con el estilo Santa Ana, una colección cerámica encontrada en el área de Trujillo en el occidente de Venezuela la cual tiene rasgos de las series Tocuyano y Barrancoide. Los diseños en espiral formados por incisiones de líneas anchas, modelado zoomórfico y el punto central sobre la arcilla adherida a las paredes de la vasija sugieren esta relación (compárese Angulo Valdez 1962: láminas IIIa-d, i,j y IV a-c con Rouse y Cruxent 1963: figura 16-a-d,f). Malambo también tiene algunas relaciones con el estilo La Pitia de la Península Guajira en el occidente de Venezuela. Las formas de las cabezas de las figurinas muestran un paralelo muy cercano. (Compárese Angulo Valdez 1962: lámina V d, e con Cruxent y Rouse 1958-9: láminas 16-nos. 8 y 17 no. 2).

Los patrones de subsistencia y el medio ambiente de Malambo sugieren que la forma de adaptación y la explotación del medio ambiente aquí es similar a la sugerida para Momil I. Las evidencias de una economía ribereña con tubérculos, suplementada con recursos reptílicos se encontraron en ambos sitios. Aún más, hay algunas similitudes en el conjunto del material, i.e. formas de la cerámica, incisiones de líneas anchas, espirales incisos y modelado biomorfo en forma de apéndices en las vasijas. Sin embargo, los modelados biomorfos de Malambo parecen ser un poquito más complejos que los de Momil. (Compárese Angulo Valdez 1962: láminas IV a-h, V-f-j con Reichel-Dolmatoff G. y A. 1956: figura 13).

Por otra parte, Ciénaga de Oro y Malambo presentan un número de paralelismos que pueden tener algún significado. En ambas colecciones se acostumbra el uso de espirales entrelazados así como un marcado uso de la decoración incisa, incisiones de líneas anchas y una alta frecuencia de adornos biomorfos modelados sobre las vasijas proyectándose hacia arriba y sobre afuera sobre los bordes (especialmente cabezas de aves). Los adornos altamente estilizados, ojos con rayas o protuberancia, formas semi-esféricas con puntos perforados en el centro y decoraciones en appli$q u e ́$ son también características de ambas colecciones.
Malambo puede representar la persistencia de una agricultura de tua lo largo de la costa norte de Colombia en (a) economías de subel primer sistencia pueden ser explicadas en

Lasencia de cerámica pintada en Malambo también se puede exde difusión estaban probablemente más haplicar debido a que las rutas de difusion estaban probatales incia el sur a lo largo de un eje este-oeste, siguiendo las rutas haber terminado antes de teriores. Todo esto sugiere que Momil I pudo haber terminado antes de la ocupación de Malambo. La aparente ausencia en Mácultura de maíz) pue-

cruciales en Momil II (i.e. cerámica pintada y agricultur
den ser explicadas en términos de una difusión desviada.

\section{OTROS SITIOS}

Monagrillo (2000 a. de C.) reportado por Willey y McGimsey (1954) consiste de una serie de lentes de conchas depositados a lo largo de lo iue costanera seca. (Véase mapa). Siendo un sitio de artes típicas y de la base de agriculmontículos de conchas, Ciénaga de Oro y Malambo. El tura de subsistencia atribuida a Momil, Cienaga de arena sugiere alguna complejo cerámico de vasijas con desgrasante de aro de otra forma son sofisticación en la decoración de diseños incisos pero de otra forma son muy simples. Las áreas excisas estaban combinadas con incisión e incisión no neas, terminando a menudo en un punto. La puntuación e incision elementos son técnicas mayores de decoración. Los remolinos, espirales, ético. Sin entrelazados y áreas excisas sugieren algo de un desarrollo estético. Sioembargo, no existe cerámica pintada y nón con nes plásticas (appliqué, cuñeado,

carrizos, adornos, asas o soportes).

Milas para el desarrollo

otra parte, puede ofrecer algunas pistas para con su apotemprano de la cerámica de Monagrillo. Momil Incisa a algunas de las cegeo en Momil IB (véase diagrama 2) se parece mucho a

8 En Momil la agricultara de cristo. Una posible ruta para la dispersión de la agricultura de maíz mienzo del primer milenio antes de Clanuras costeras del sistema del Río Magdalena replegandose más hacia el sur a través de la cuenca del rio San Jorge y hacia el Magdalena medio, siguiendo el rio Cesa la Siecia $\mathrm{La} \mathrm{Paz}$ (una comunidad mestiza moderna un poquito al sureste de cia La Paz (una comunidad mesta no poquito más arriba de Manaure ha sido una ruta principal de comercio
rra de Perijá. El paso situado un pocial. y movimiento desde tiempos tempranos. Esta ruta provee un acceso fácil al Lago Mancho Peludo, río aba. mente al sistema del río Guasare, a lo largo del cual se encuentra el sitio de Racticada en las regiones jo. Hay evidencias, como se mencionó antes, que la agricultura de maiz fue practicada. Sin embargo, la del Cesar y Ranchería y en Rancho Peludo durante el primer milent) ha documentado la existencia tannaturaleza precisa de la difusión del maíz no es clara. Zucchí en La Betania en los Llanos del Orinoto de budares como del complejo lítico de manos y metates en sugiere una inversión en la popularidad del co. Las manos y metates aparecen antes que los budares lo cuarece alrededor de los comienzos del primer complejo anterior. Zucchi sugiere que el complejo litico aparece alrededor de lítica, figuras 140-145 para milenio despues de Cristo. ýána 231 para las fichas de carbono -14). 
1956: lámina VII 8, 10-12 con Willey y McGimsey 1954: figura 46a-b) 1956: lamina VII 8, 10-12 con Willey y McGimsey 1954: farigua AppliAdemás, la siguiente tradición ceramica en Monagrillo, Saricá qué tiene mucha similitud con Momil Modelada Aplicada, (compá rese Willey y McGimsey 1954: figura 48-s con Reichel-Dolmatoff, G. y A 1956: $175-6$, lámina X $3,5,7$, y 8). El hachurado [fine-line hatching]cruzaen ambos sitios.

Canapote, una serie de montos de conchas con fechor de 2000 a. de C. (Reichel-Dolmatoff, G. 1965b: 58), es contemporáneo dor de vecon Monagrillo. El desgrasante de arás temprano: Puerto Hormiga. Bisgetal, es característico del Complejo más temprano. Puerto Hormiga. Bishof sugiere que existe similitudes entre Monagrillo y Canapote, el más antiguo de los dos niveles debajo del nivel cultural de Barlovento en $\mathrm{Ca}$ napote y este último [Canapote] puede llenar el vacío cronológico entre Puerto Hormiga y Malambo (1966:489). Willey piensa que algunos de los materiales de Barlovento son similares al estilo cerámico de Monagrillo. Véase Reichel-Dolmatoff, G. 1956:267). Esto es muy significativo ya que el desgrasante de arena ciertamente representa una sofisticación en la técnica de manufactura cerámica.

Monagrillo también tiene algunas afinidades con los diseños espirales onchos Barrancoides y el uso de líneas incisas con puntos termina les (Willey y McGimsey 1954:131). El complejo Monagrillo no es una in les (Willey dustria cerámica incipiente pero representa claramente una difusión periférica de una tradición cerámica ricamente dotada la cual se estaba haciendo sentir en las llanuras costeras del norte y en los tributarios de ciendo dercer milenio antes de Cristo. Sur América durante la ultima parte del tercer en Momil y probablemenEsta tradición estaba fuertemente representada en Momil y probablemente forma la base del complejo puro de Ciénaga de Oro. Ella tambien tuvo un impacto en las culturas concheras de las regiones costaneras de Colombia y Panamá.

Hay evidencia para la interrelación de esas secuencias en la técnien conapote como en ca de Momil IB. Es diseños curvilineales con en Momil desde los niveles más tempranos y los diseños curvilineales con estampado dentado de Barlovento tienen afinidades con Momil II. (Compárese Bishof 1966: figura 3-4,8,9,11,12 con Reichel-Dolmatoff G. y A. 1956: lámina XVI $3,4,10$ ). Aún más, Bishof (comunicación personal) ha 1956: lámina XVI 3,4,10) An la superficie del sitio de Bocachica en encontrado material Momil en la sugiriendo contactos entre los agricul, Cartagena, al noreste concheros.

Las técnicas decorativas, formas de vasijas, junto con un medio amiente similar de los sitios costeros arriba discutidos, sugieren que alguna biente similar de los sitios costeros arriba discutidos, sugieren que alguna relación existió entre Monagrillo y el Complejo Canapote-Barlovento. Aún más, es muy tentador ver ambas regiones bajo una influencia de una tradición momiloide.

Valdivia (2500-2000 a. de C.) fue probablemente contemporánea con $16^{M}$ elaborado (Ladd 1964: 227). En Valdivia encontramos una gama de tipos cerámicos, superficies de la cerámica bañada y decoración plástica. Los materiales incisos de Valdivia son distintivos y no se aproximan a los diseños curvilineales que dominan el tipo cerámico Monagrillo Inciso. Además, la colección de Valdivia contiene numerosas figurinas, orejeras, punzones y conchas perforadas. Mientras que tanto Monagrillo como Valdivia representan adaptaciones costaneras, la última es muy compleja para haber subsistido basada solo en una economía marina.

Valdivia comparte un número de rasgos con Momil. Ollas con bordes evertidos son las formas más numerosas de vasijas que se encuentran en ambos sitios desde los niveles más tempranos. El trono del jaguar es también común en ambas tradiciones. (Compárese Reichel-Dolmatoff, G. y A. 1956: lámina XXII con Meggers, Evans y Estrada 1965: figura 63)

La banda appliqué de Valdivia se parece mucho a Momil Dentada Crestada y Momil Acanalada Aplicada. (Compárese Meggers, Evans y Estrada 1965: lámina 27-28, con Reichel-Dolmatoff, G. y A. 1956: lámina VIII 1-3, y lámina VI 3, 5-7 ).

La decoración línea fina incisa de Valdivia y Machalilla Ayangue Inciso que se encuentra en los boles carinados, es idéntica a algunos de los diseños de Momil Negra Incisa (nivel IB) que se encuentran en los boles y discos (véase figura 5 y compárese Meggers, Evans y Estrada 1965: láminas 63 o, q y 131 j, k con Reicheld-Dolmatoff, G. y A. 1956: lámina VII $6,7,9)^{9}$

También existen paralelos entre Valdivia Modelada y Momil Aplicada Simple (compárese Meggers, Evans y Estrada 1965: lámina 78 con Reichel-Dolmatoff G. y A. 1956: lámina VI 8) y las vasijas carinadas en ambos sitios son similares en forma. (Compárese Meggers, Evans y Estrada 1965: figura 54-nos. 8,9 con Reichel-Dolmatoff G. y A. 1956: figura 15).

\section{CONCLUSION}

El material antes presentado sugiere que un examen de los sitios locales y secuencias adyacentes en el noroeste de Sur América pueden dar luz en la interpretación y fechamiento de la secuencia de Momil. Con la definición de la naturaleza del complejo cerámico de Ciénaga de Oro se delimitó un complejo intruso.

La existencia en Ciénaga de Oro de esos dos complejos implica que se requirió un largo período para que esas tecrologías cerámicas distintivas de agricultores de Selva Tropical se diversificaran y después convergieran espacialmente en el bajo río Sinú probablemente bajo el control de un solo grupo étnico. Se ha hipotetizado que la ocupación original en Ciénaga de Oro posdata Momil I y probablemente fue coexistente con los primeros niveles de Momil II (nivel 6). Aún más, tanto Momil I como

9 Cerámicas parecidas a Machalilla (ayangue Inciso) se han descubierto recientemente en el Bajo 9 Cerámicas parecidas a Machalilla (ayangue Inciso) se han descubie como se pensaba, sino que es taba bien desarrollada en las Tierras Bajas del Caribe de Colombia (Reichel-Dolmatoff, G.n. d.: 17)" 
Ciénaga de Oro son productos de una expansión temprana de la Cultura de Selva Tropical paralela al estilo Saladoide del este de Venezuela mientras que el complejo intruso en Ciénaga de Oro es parte de una ramificación tardía de la expansión Barrancoide ${ }^{10}$.

Barrancas, la más temprana de la expansión Barrancoide identificada por Cruxent y Rouse, comenzó a difundirse en el bajo Orinoco tan temprano como 900 a. de C. (1958-9: cuadros 2,3). El Palito, el cual es el prano mente en 400 a. de C. (Rouse y Cruxent 1963: 82-84). Debido a las fuertes relaciones entre los materiales intrusivos en Ciénaga de Oro y el estilo Barrancas representado por el Palito, una fecha de 300 a. de C. parece apropiada para la ocupación final en Ciénaga de Oro. Si este es el cace apropiada para la ocupación final en Cienaga de Oro. Si este es el caso entonces la secuencia entera de Momil ha seguido su curso hasta siglo segundo de la era Cristiana ${ }^{11}$ El sitio nunca fue reocupado pero fue usado en fechas posteriores como cementerio durante la dominación Caribe en las llanuras costeras del norte de Colombia. Los restos culturales intrusivos de Betanci hallados en los niveles superiores de Momil (Reichel-Dolmatoff, G. y A. 1957: 138-140) probablemente datan de 1000 después de Cristo.

CRONOLOGIA PROPUESTA

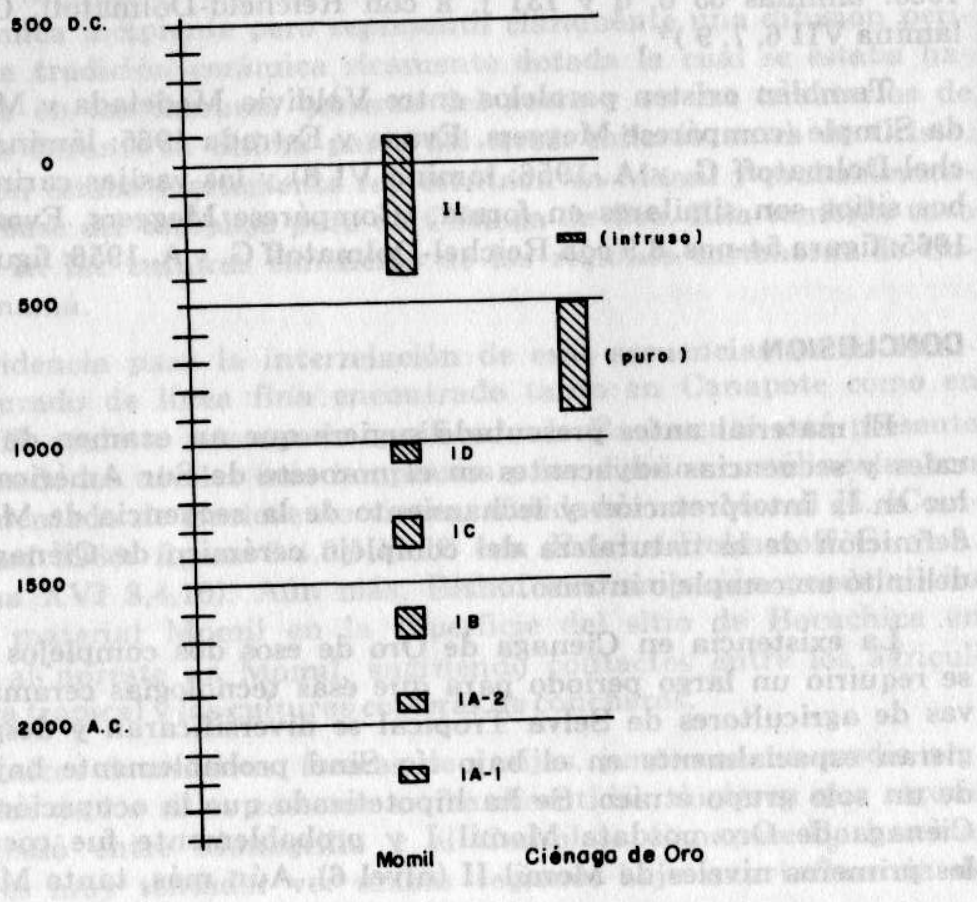

10 Rouse y Cruxent (1963) proponen el mismo tipo de desarrollo complejo para Venezuela.

11 Henning Bishof (1966:490) sugiere que en Canapote hubo una tradición parecida a la Barrancoide.
Se ha intentado colocar el arribo de la agricultura de Selva Tropical al norte de Colombia en una cronología unilineal (Ford 1969; ReichelDolmatoff 1965b; Willey 1958). Esto está evidenciado en la secuencia que coloca Puerto Hormiga, Canapote, Barlovento, Zambrano y Malambo antes que Momil I y II. También está evidenciado por la colección de Ciénaga de Oro después de Momil II hecha por los Reichel-Dolmatoff (1957: 128). El argumento aquí expuesto es que puesto que Momil y Ciénaga de Oro, tanto en su subsistencia como en su cultura material, fueron tan diferentes de las culturas costeras menos desarrolladas de montículos de conchas, difícilmente ellos han podido ser contemporáneos con éstas.

Si como se sugiere en este informe, Momil data de un período contemporáneo con las culturas concheras, entonces las diferencias tecnológicas, formales y estilísticas entre esos dos debe explicarse. La co-existencia de dos tradiciones cerámicas diferentes pueden explicar esta aparente contradicción. Este punto de vista que ve una forma de vida arcaica persistiendo hasta tiempos más "modernos" e influida por contactos de variable intensidad por grupos del interior más avanzados tecnológicamente, puede ser una explicación más fructífera para todo el desarrollo cultural de esta región ${ }^{12}$ Esto permitiría un mejor esquema de trabajo para interpretar la creciente cantidad de evidencia arqueológica proveniente de Colombia y áreas aledañas. De cualquier forma, los Reichel-Dolmatoff prontamente admiten que las tradiciones representadas en Momil no son "aislamientos locales" sino se trata evidentemente de un horizonte muy amplio que contiene ciertos estilos cerámicos característicos, con una situación ecológica persistentemente similar (1957:127).

El modelo presentado en este informe apoya al propuesto por Lathrap (1970) con relación a la expansión de grupos de Selva Tropical durante el período $3000-0$ a. de C. La existencia de gentes cuya tecnología cerámica estuvo tan altamente desarrollada en esta época, sugiere que los agricultores de Selva Tropical tuvieron una historia larga y elaborada fechable bien dentro del tercer milenio antes de Cristo en las tierras bajas de Sur América. Se ha propuesto un fechamiento para la secuencia de Momil y su asociación con otras secuencias en el norte de Sur América, no como una solución definitiva a los problemas cronológicos presentados, sino como una indicación que esos problemas han sido ignorados muy a menudo por los arqueólogos interesados en el perfil general de la historia cultural del Nuevo Mundo.

12 En un informe reciente, Gerardo Reichel-Dolmatoff presenta un resumen al día de la cronología ar. queológica Colombiana en el cual postula una fecha más temprana para la secuencia Momil y la co-exis. tencia de una tradición de montículos de concha en la costa y una tradición de aldeas con agricultura de tubérculos en la llanura costera del norte de Colombia durante el segundo milenio antes de Cristo (n.d. 
ANGULO VALDEZ C.

1962 "Evidencias de la Serie Barrancoide en el Norte de Colombia". Revista Colombiana de Antropología. 11: 73-88.

BISHOF, $\mathrm{H}$

1966 "Canapote, An Early Ceramic Site in Northern Colombia: Preliminary Site Report". 36th International Congress of Americanists, 1:483-491

CRUXENT, J.M. e IRVING ROUSE

1958-9 An Archaeological Chronology of Venezuela. Pan American Union, Social Science Monographs No. 6, 2 volumes.

FORD, J

LADD, $J$ 1964

"A Comparison of Formative Cultures in the Americas". Smithsonian Contributions to Anthropology, 11 .

"Archaeological Investigations in the Parita and Santa Maria zones of Panama". Bureau of American Ethnology, Bulletin 193 .

LATHRAP, D.W

1958

"Review of Momil: Excavaciones en el Rio Sinu by Gerardo and Alicia Reichel-Dolmatoff". American Journal of Ar. chaeology, 62: 360-362

1966 Colombia Prehistory Comes of Age. Science 152: 923-925.

1968

Aboriginal Occupation and Changes in River Channel on the Central Ucayali, Peru, American Antiquity, 33 (1): 62-79.

1970 The Upper Amazon. New York: Praeger Books.

MEGGERS, B.

1954

Environmental Limitations on the Development of Culure. American Anthropologist, 56:801-824.

MEGGERS, B. y C. Evans

1957 "Archaeological Investigations at the Mouth of the Amazon". Bureau of American Ethnology, Bulletin 167.

MEGGERS, B., C. Evans y E. Estrada

1965 Early Formative Period of Coastal Ecuador: The Valdivia and Machalilla Phases. Smithsonian Contributions to Anthropology, 1 .

REICHEL-DOLMATOFF, G.

1954

A preliminary Study of Space and Times Perspective in Northern Colombia. American Antiquity, 19: 352-366.

1955 "Excavaciones en los Conchales de la Costa de Barloven to". Revista Colombiana de Antropología, 4: 249-272.

1965 a Enes Arqueologicas en Puerto Hormiga (Depto. de Bolivar). Ediciones de la Universidad de los Andes, Bogotá, Antropológica No. 2.

REICHEL-DOLMATOFF, G.

1965b Colombia. London: Thames and Hudson.

n. d. "The Archaeological Chronology of Colombia". Instituto Coombiano de Antropología, (manuscript) Bogota.

REICHEL-DOLMATOFF, G. y A.

1951 "Investigaciones Arqueológicas en el Depto. del Magdalena, Colombia". Boletín de Arqueología, 3 (1-6).

1956 “Momil, Excavaciones en el Río Sinú”. Revista Colombiana de Antropologia, 5: 109-334.

1957 "Reconocimiento Arqueológico de la Hoya del Río Sinú". Revista Colombiana de Antropologia, 6: 31-149.

1961

"Investigaciones Arqueológicas en la Costa Pacífica de "Colomian 10:237-330.

ROUSE IRVING y J. M. CRUXENT

1963

Venezuelan Archaeology. New Haven. Yale University.

ROWE, J. H.

1961

Stratigraphy and Seriation. American Antiquity, 26: 324-330.

SAUER, $\mathrm{C}$.

1952

American Geographical Society

WILLEY, G. R.

1958

Estimated Correlations and Dating of South and Central American Culture Sequences. American Antiquity, 23: 353-378.

WILLEY, G. R. y C. McGimse

The Monagrillo culture of Panama. Harvard University Peabody Museum of Archaeology and Ethnology, 49 (2).

ZUCCHI, A

1967

Betania: Un Yacimiento Aruqeológico del Occidente de la Universidad Central de Venezuela, Ph. D. Dissertation.

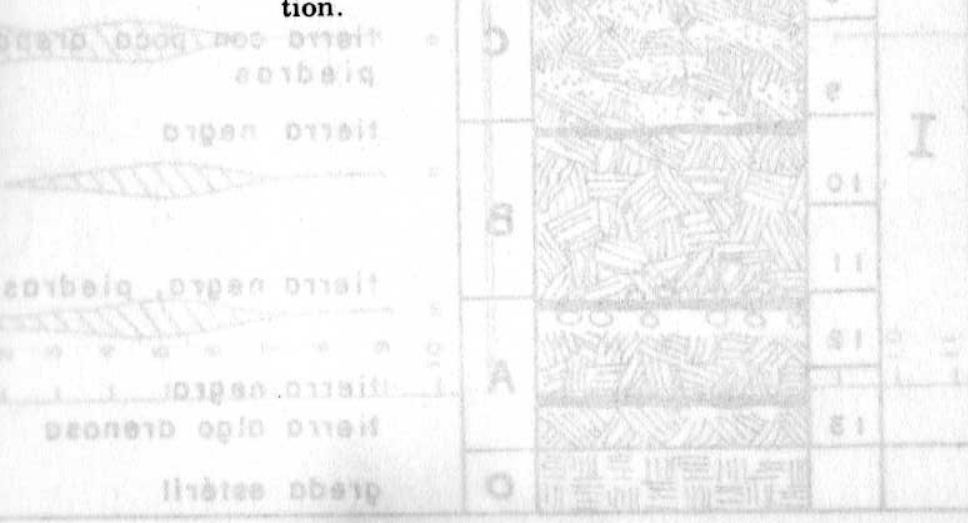

azer A $x, 0$ athotomiog - ienatak iab 


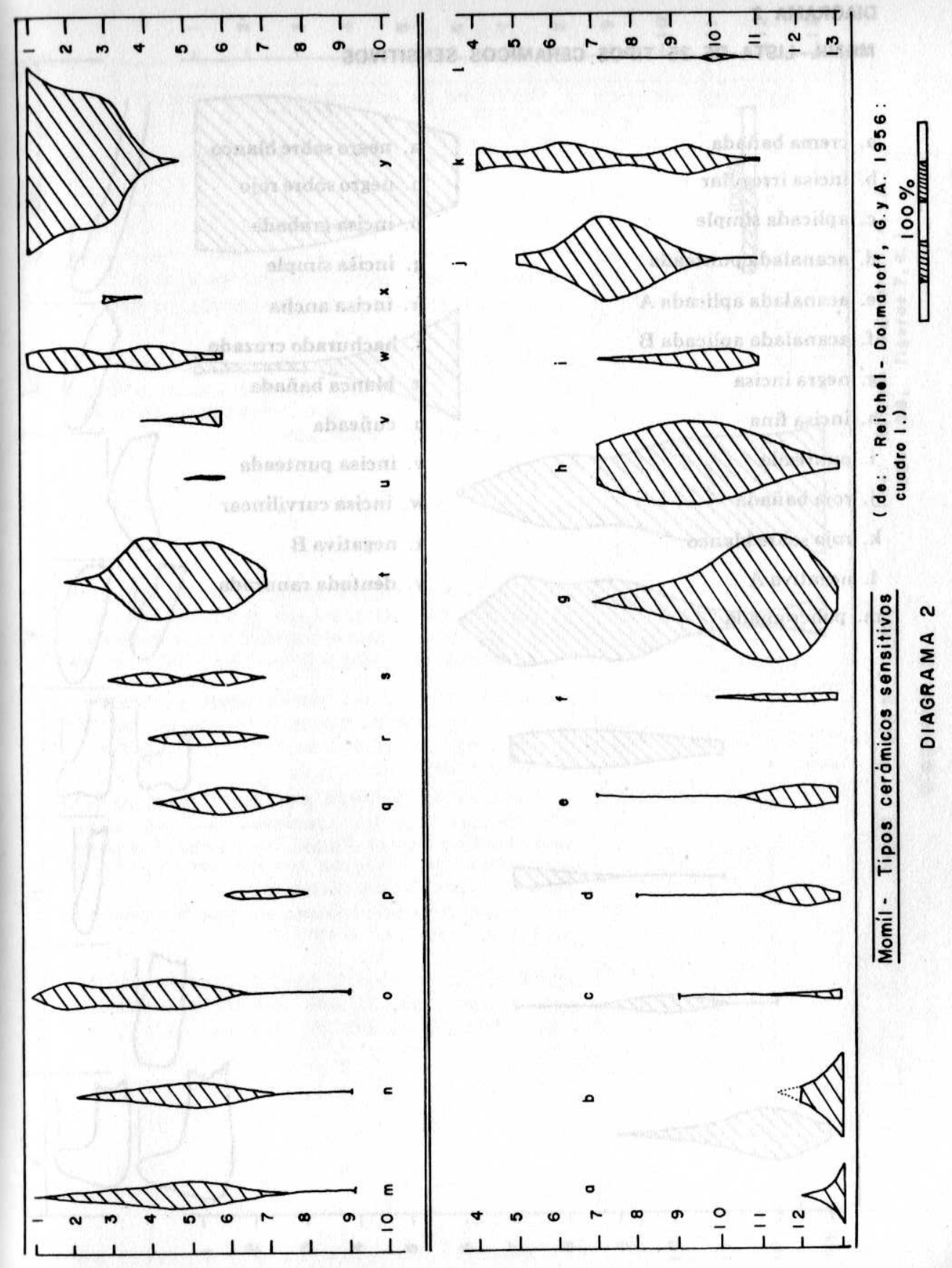

tierro negra

tierra algo arenoso

greda estéril

(de: Reichel-Dolmatoff, G. y A. 1956:123. 


\section{DIAGRAMA 2}

MOMIL-LISTA DE 25 TIPOS CERAMICOS SENSITIVOS

a. crema bañada

b. incisa irregular

n. negro sobre blanco

o. negro sobre rojo

p. incisa grabada

q. incisa simple

r. incisa ancha

s. hachurado cruzado

t. blanca bañada

u. cuñeada

v. incisa punteada

w. incisa curvilinear

x. negativa $B$

y. dentada ranurada k. rojo sobre blanco

1. negativa $A$

m. policromada

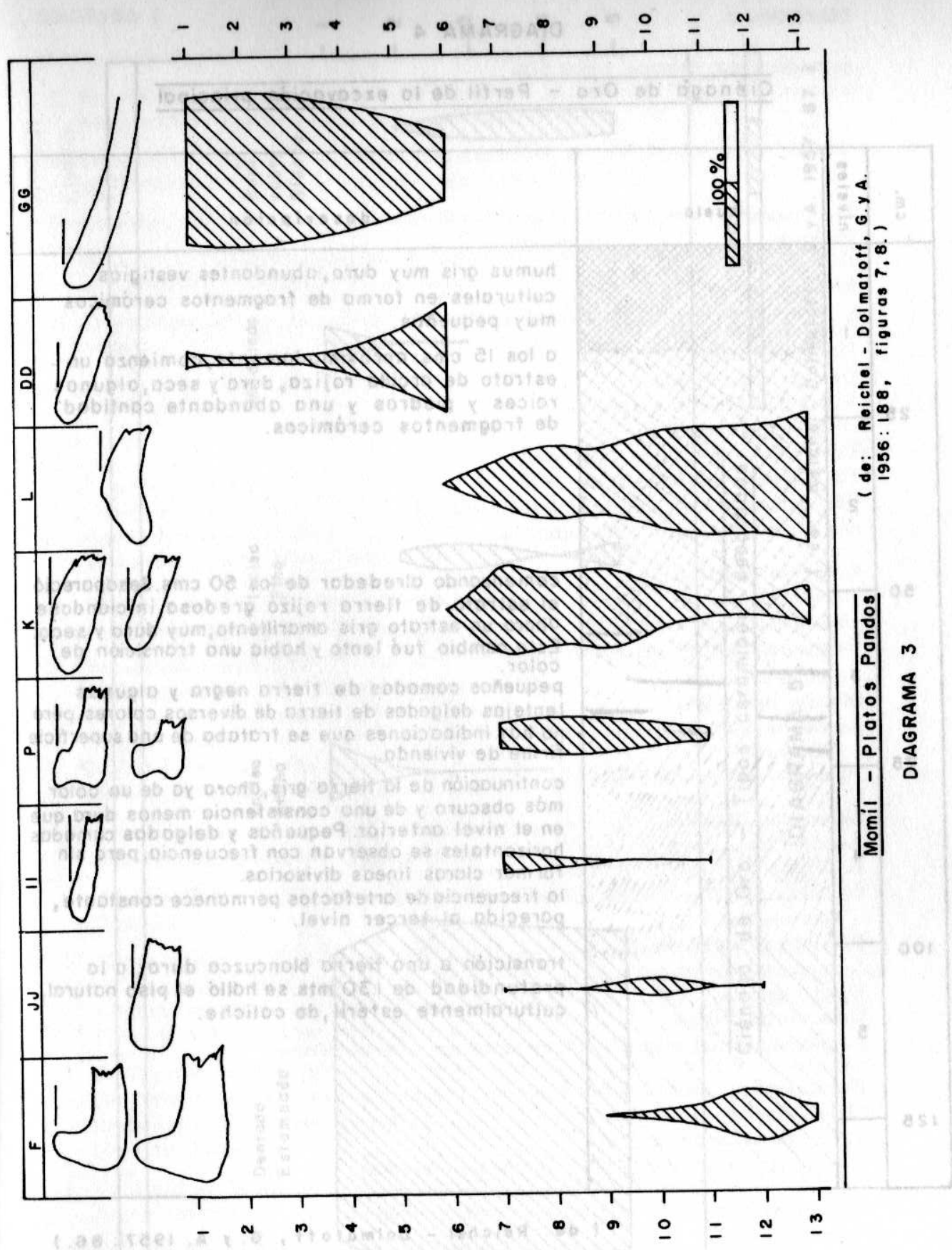




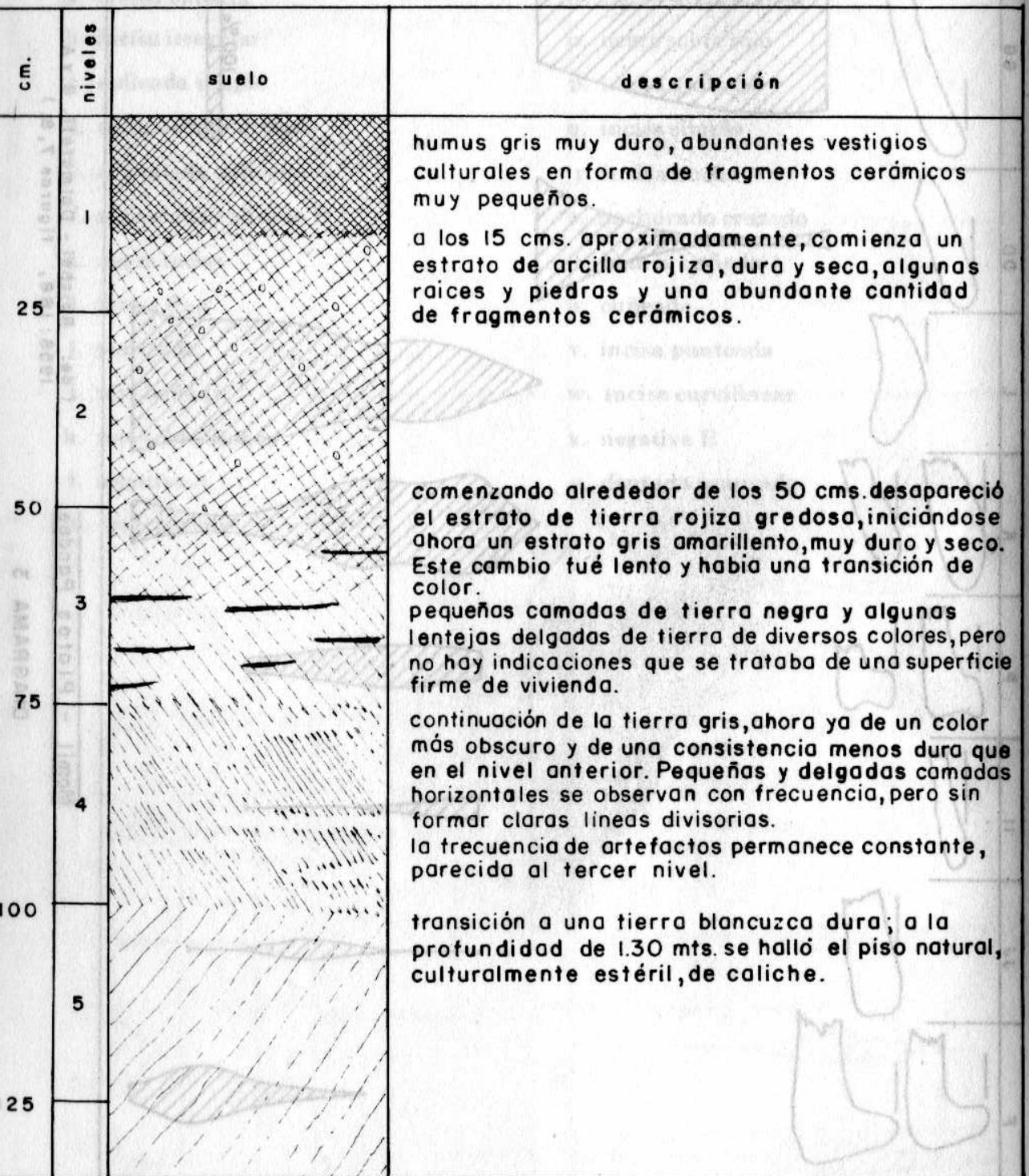

(de: Reichel - Dolmotoff, G. y A. 1957:86.)
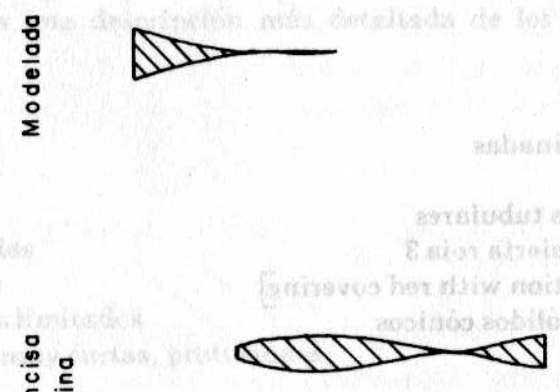

드 든
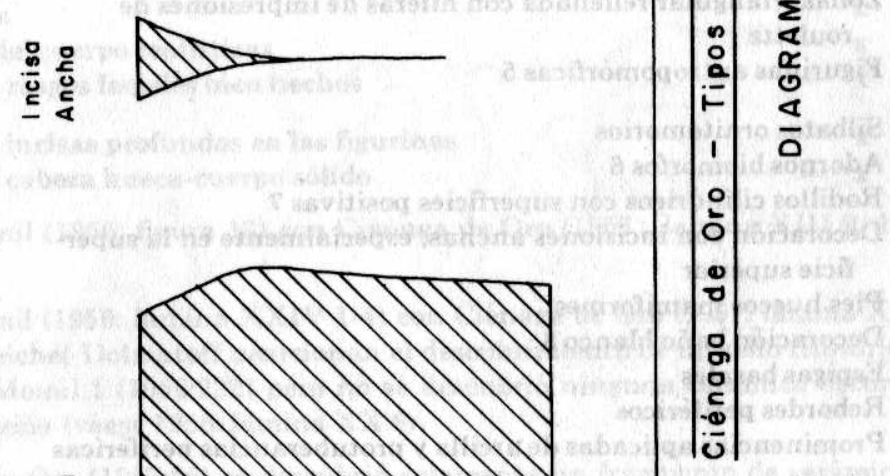
Industria sílex

Budares

Crema bañada

Sellos planos

Sonajeras [rattles] 1

Discos de arcilla

Corrugación aplicada 2

Vasijas fuertemente carinadas

Decoración incisa fina

Picos tubulares u objetos tubulares

Pintura bicroma con cubierta roja 3

Bichrome paint decoration with red covering

Vasijas con pies cortos sólidos cónicos

\section{Cerámica miniatura}

Bases múltiples

Decoración roulette dentado

Banda roja pintada en borde, especialmente en platos pandos

Volantes de huso 4

Motivos sigmoideos

Zonas triangular rellenada con hileras de impresiones de roulette

Figurinas antropomórficas 5

Silbatos ornitomorios

Adornos biomorfos 6

Rodillos cilíndricos con superficies positivas 7

Decoración con incisiones anchas, especialmente en la super-

ficie superior

Pies huecos mamiformes

Decoración baño blanco 8

Espigas basales

Rebordes periféricos

Prominencias aplicadas de arcilla y protuberancias periféricas

Floreros con reborde basal 9

Manos y metates 10

Vasijas pintadas polícromas

Pintura bicroma negra sobre blanco, negro sobre rojo

Pigmento blanco usado para rellenar incisiones anchas 11 Bases anulares

Zonas de decoración delimitada

boles pandos con patas tubulares largas

Decoración interna en floreros pandos

Bordes anchos con lóbulos modelados o triángulos proyectados
1. Reichel-Dolmatoff, G. y A. 1957:80, lámina X 18 describieron el hallazgo superficial en Maracayo, un complejo de Ciénaga de Oro.

2. Compare Ciénaga de Oro (Reichel-Dolmatoff G. y A. 1957: lámina 19) con Momil (Reichel-Dolmatoff G. y A. 1956: lámina VI 7 y VIII 1,3).

3. Compare Momil Rojo sobre Blanco con Ciénaga de Oro Rojo sobre Ocre.

4. Compare el hallazgo superficial en Maracayo (1957:82) con las descripciones de los hallazgos de Momil IB y II (1956:217).

5. El siguiente es una descripción más detallada de los rasgos selectos de las figurinas:

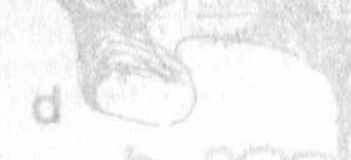

Figurinas sólidas

Cabeza grande

Rasgos faciales limitados

Extremidades muy cortas, protusiones

Sexo indicado solamente por los senos

Figurinas sentadas y paradas

Patas gordas mamiformes y huecas

(hallazgo superficial en Ciénaga de Oro)

Perforaciones horizontales en las figurina

Formas huecas

Proporciones del cuerpo realísticas

Detalles de los rasgos faciales bien hechos

Decoraciones incisas profundas en las figurinas

Combinación: cabeza hueca-cuerpo sólido

6. Compare Momil (1956: figura 13) con Ciénaga de Oro (1957: lámina XIII 9, 12. 13, y 15).

7. Compare Momil (1956: lámina XXIV 1-4) con Ciénaga de Oro (1957: lámina XII 11-14). Los Reichel-Dolmatoff mencionan el descubrimiento de un sello cilíndrico completo en Momil I (1956:222) pero no se descubrió ninguna cerámica decorada con este diseño (véase 1956: lámina XX 8).

8. En Ciénaga de Oro (1957:96) se descubrió solamente un fragmento de cerámica. Este mostró un posible baño blanco el cual podría relacionarse con Momil Blanca Bañada el cual aparece por primera vez en los niveles más bajos de Momil II (véase diagrama 2).

9. Un perfil de borde probablemente de un reborde basal de Ciénaga de Oro es diagramado pero no descrito (1957: figura $5-\mathrm{V}$ ).

10. Una posible piedra de moler fue encontrada en el nivel 2 en Ciénaga de Oro (1957:98). Fue hecha de piedra arenisca y fácilmente podría representar un desarrollo incipiente de la preparación de semilla.

11. Se hace referencia a Momil Negra Incisa y Negra Dentada Fina, descrita en el informe de Momil (véase 1956:146,176) 


\section{figura 1}
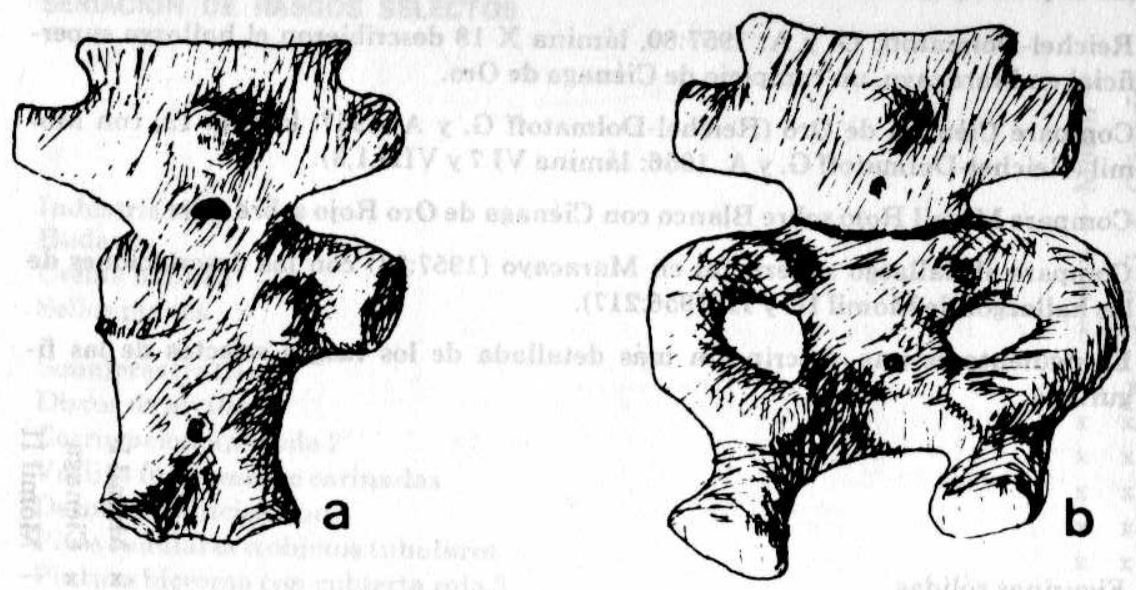

Momil I Figurinas antropomórficas

(de: Reichel-Dolmatoff, G.y A. 1956 : lámina $X X \mid I \quad, 6$.)

figura 2

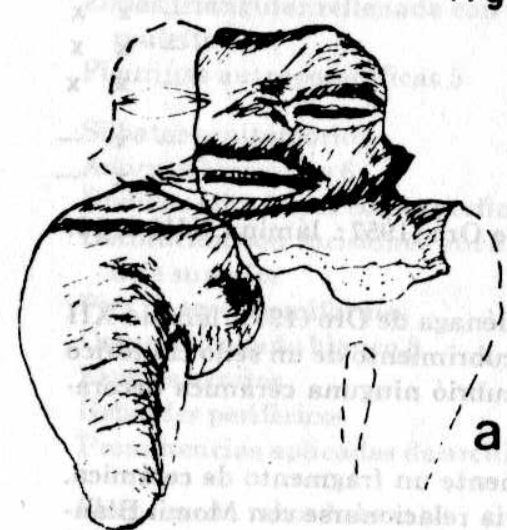

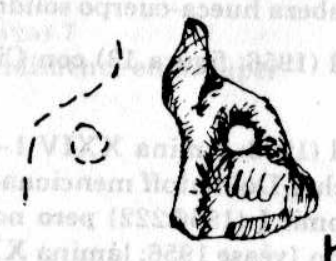

b

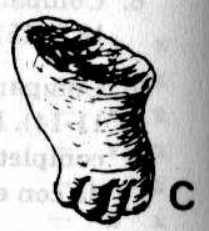

Momil II Figurinas antropomórficas

(de: Reichel-Dolmatoff, G.y A. 1956 : lámina XXIII $5,16,18$.) figura 3

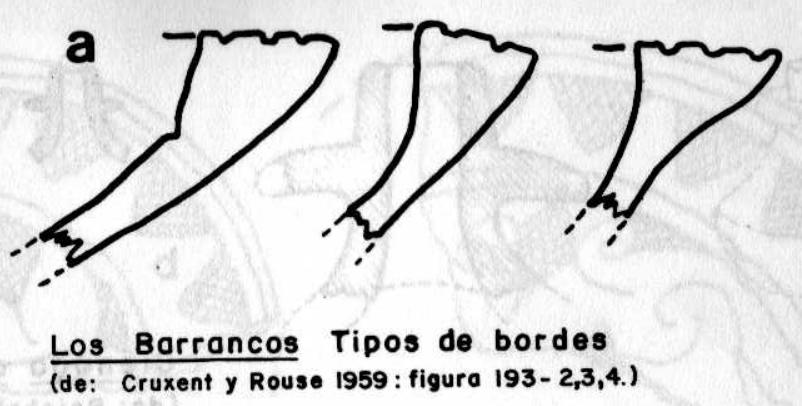

b

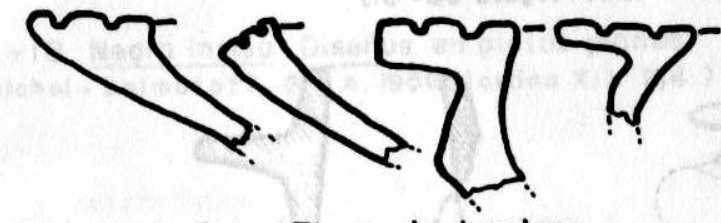

Cienoga de Oro Tipos de bordes

(de: Reichel-Dolmatoff, G.y A. 1957: figura 5-h.)

C
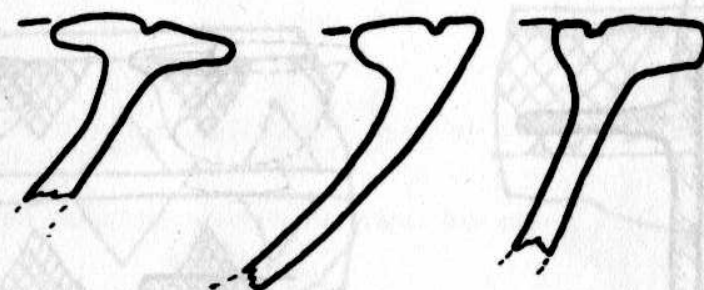

EI Polito

Tipos de bordes

(de: Cruxent y Rouse 1959 : figura $61-7,10,11$.)

d

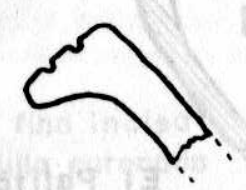

Momil I

Tipos de bordes

(de: Reichel-Dolmatoff, G.y A. 1956 : figura 9- 3,10.) 
figura 4

figura 5

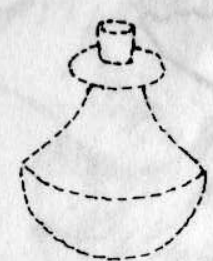

a

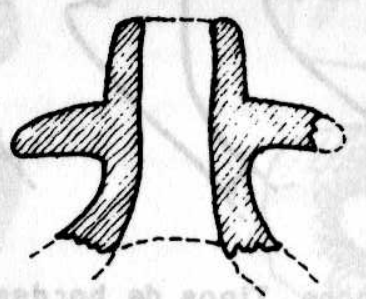

(A) s)

b

\section{Cienaga de Oro}

(do: Reichel-Dolmatof

G.y A. 1957: figura 5-R.)

\section{La Betania, Venezuela}

(de: Zucchi 1967: figura 38-3.)
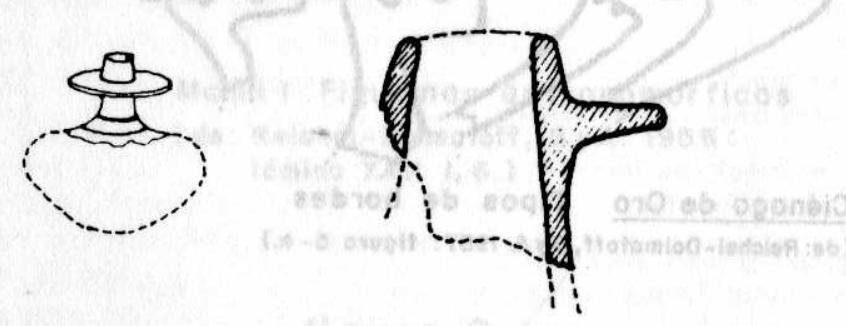

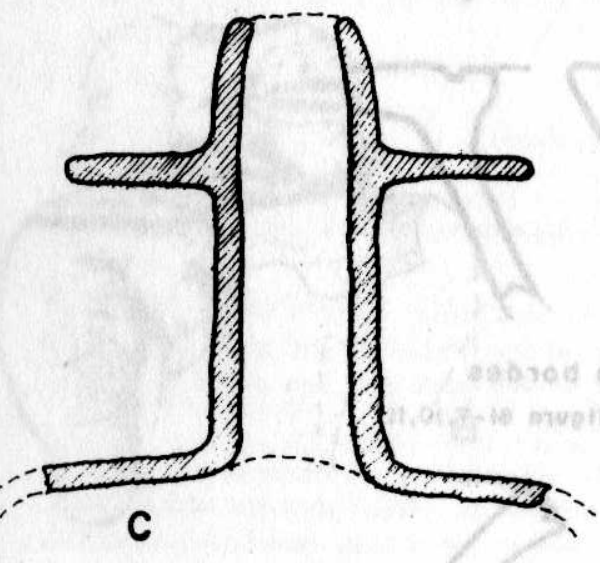

Caño del Oso, Venezuelo (de: Cruxenty Rouse 1958-9 figura $154-(b, 2$.)

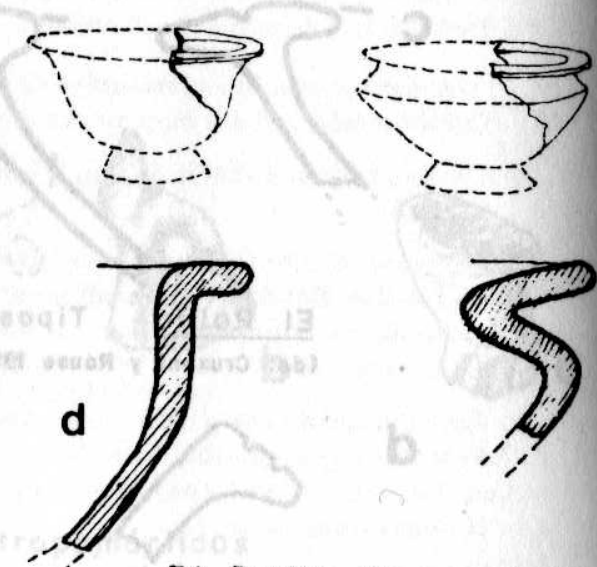

El Palito, Venezuelo (de: Cruxent y Rouse 1958-9: figura 60- 10,12 .)

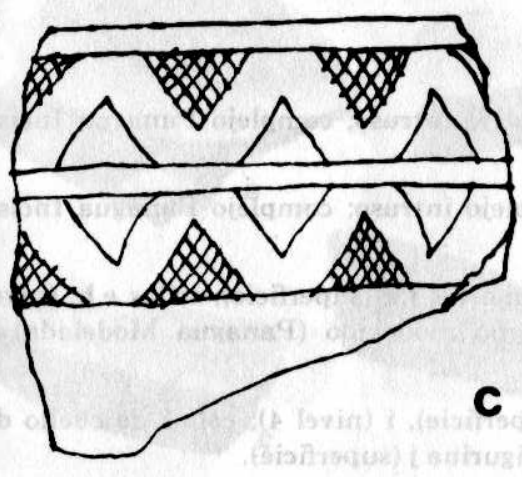

Valdivia Línea fina incisa diseño en escudillo corenado (de: Meggers, Evans y Estrada 1965 : lámina 63 p.)

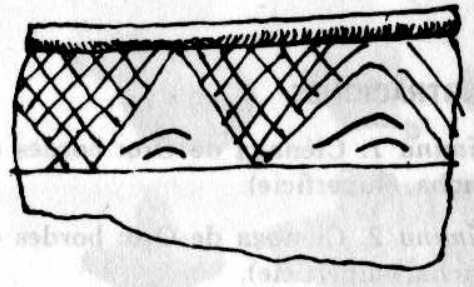

d

Machalilla Ayangue diseño en escudilla carenado (de: Meggers, Evans y Estrada (de: Meggers, Evans 

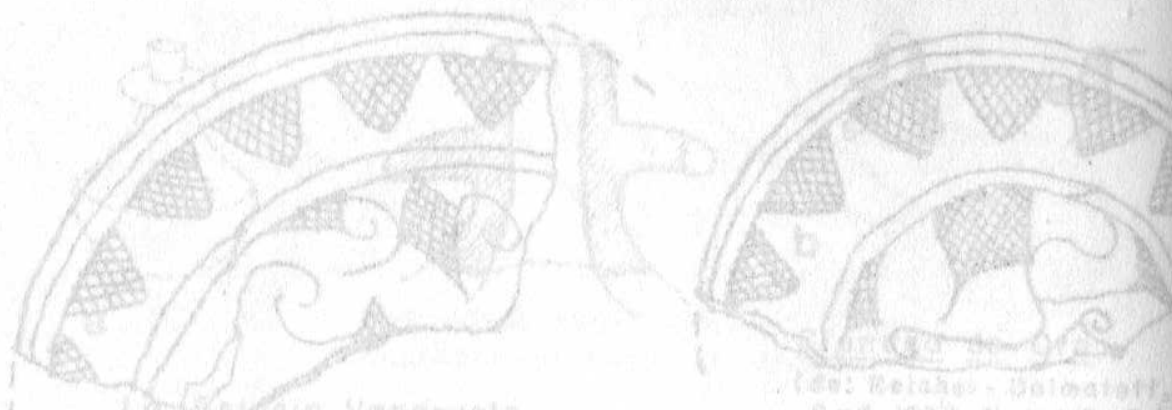

$c$

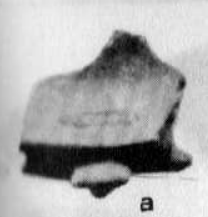<smiles>[3H][V]</smiles>

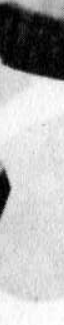
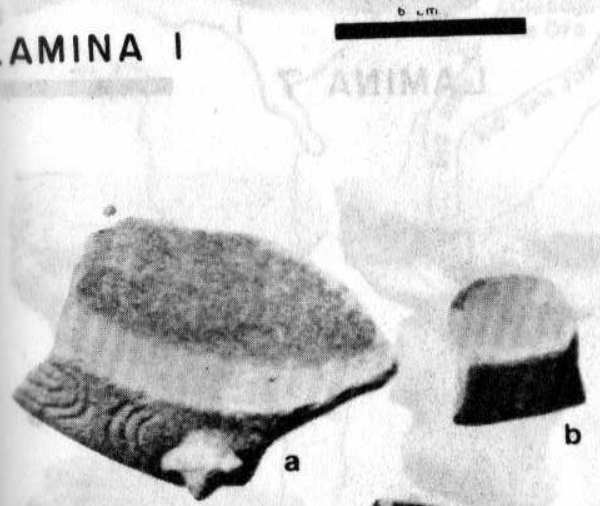

Lámina 1. Ciénaga d

Lámina 2. Ciénaga de Oro: bordes del complejo intruso; complejo Panagua Incisa Ancha, (superficie).

Lámina 3. Ciénaga de Oro: adornos antropomorfos $\mathrm{j}, \mathrm{k}$ (superficie); sellos e,h, (nivel 4), i (superficie); bordes a-c,f (nivel 4); diseño modelado (Panagua Modelada) 9 (nivel 1); florero miniatura d (nivel 4).

Lámina 4. Ciénaga de Oro: silbatos a,g. (superficie), i (nivel 4); collar de cuello de botella $\mathrm{h}$ (nivel 2); adornos b-d, $\mathbf{f}$ (superficie); figurina $\mathbf{j}$ (superficie).

Lámina 5. Momil I (diseños dentados): Dentada Zonificada a (nivel 10), b (nivel 8), c (nivel 11), d (nivel 12), e-f (nivel 8), 1 (nivel 10); Negra Dentada Fina g, i-k (nivel 11), h,n (nivel 10), m (nivel 8), o (nivel 12).

Lámina 6. Momil II: Cuñeada a (nivel 6); Momil I Dentada Zonificada bordes b.d f,h (nivel ?), c,e,i, (nivel 10), g (nivel 9).

Lámina 7. Ciénaga de Oro: Panagua Dentada Estampada a-g (nivel 3), h-k (nivel 2).

Lámina 8. Ciénaga de Oro: figurinas antropomorfas Tipo B a,e (superficie), Tipo A b-d, f-g (superficie).

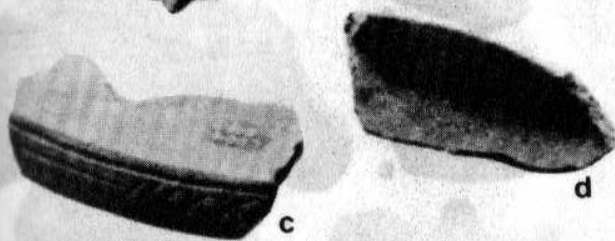

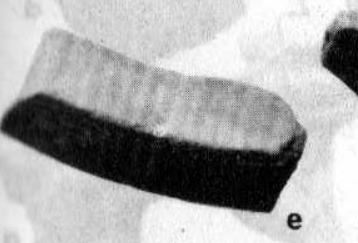

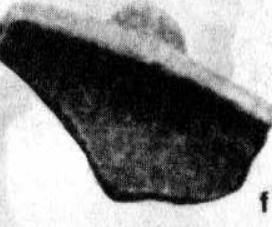

LAMINA 2

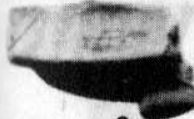
e

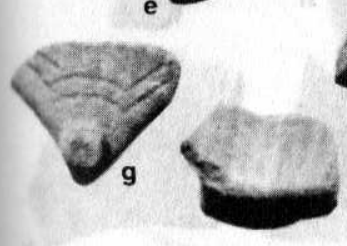

LAMINA

b
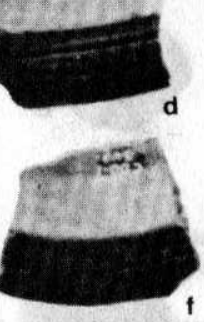

(6):
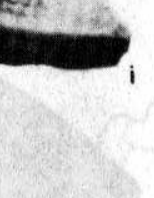

ILUSTRACIONES
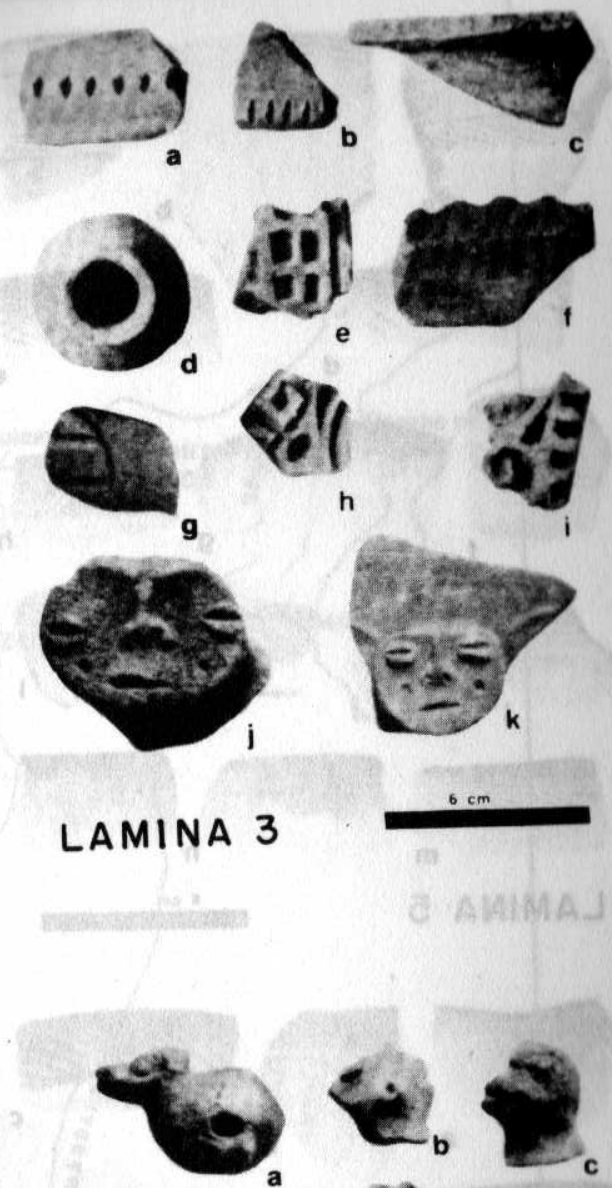


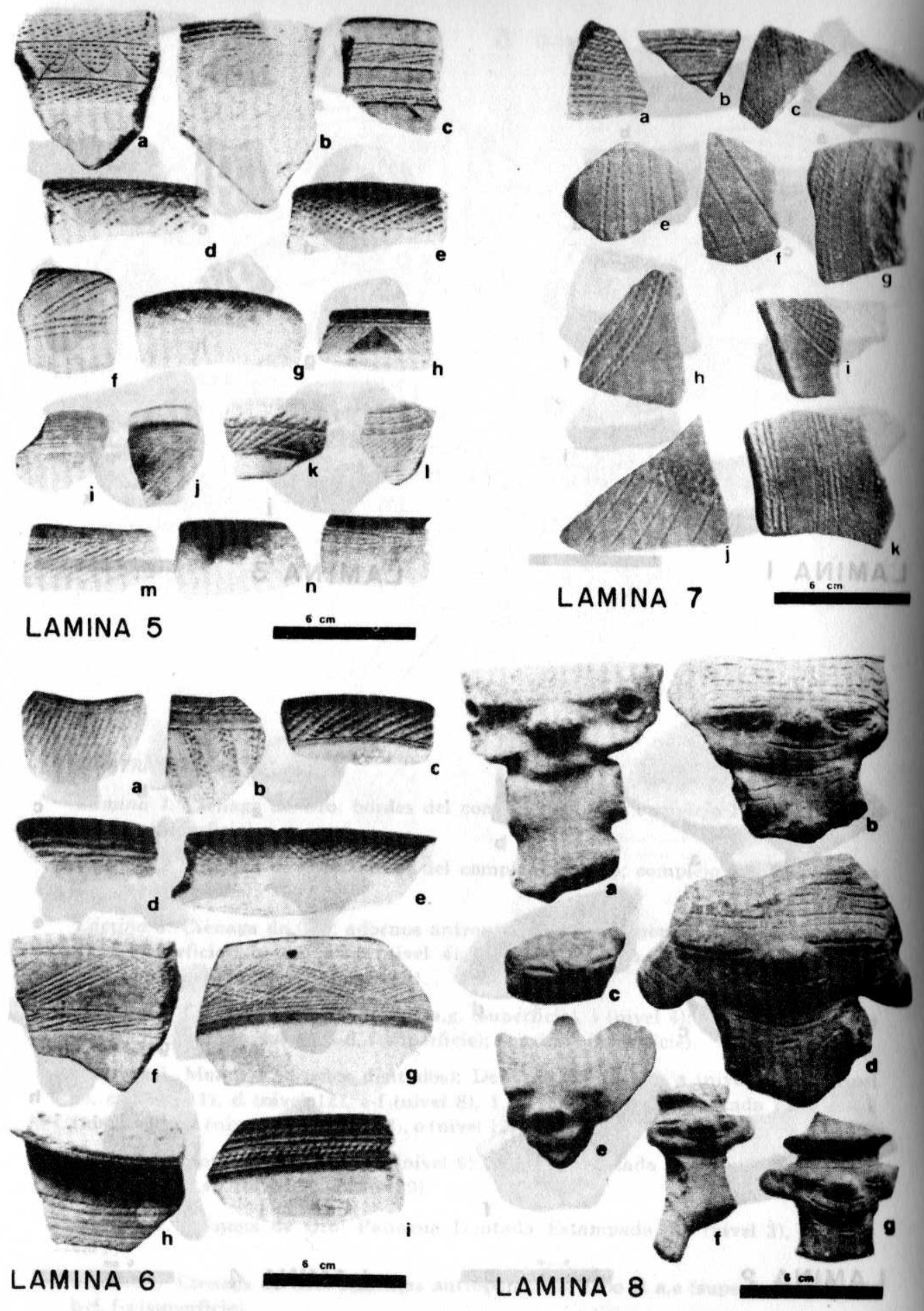

\section{MAPA}

CARIBE
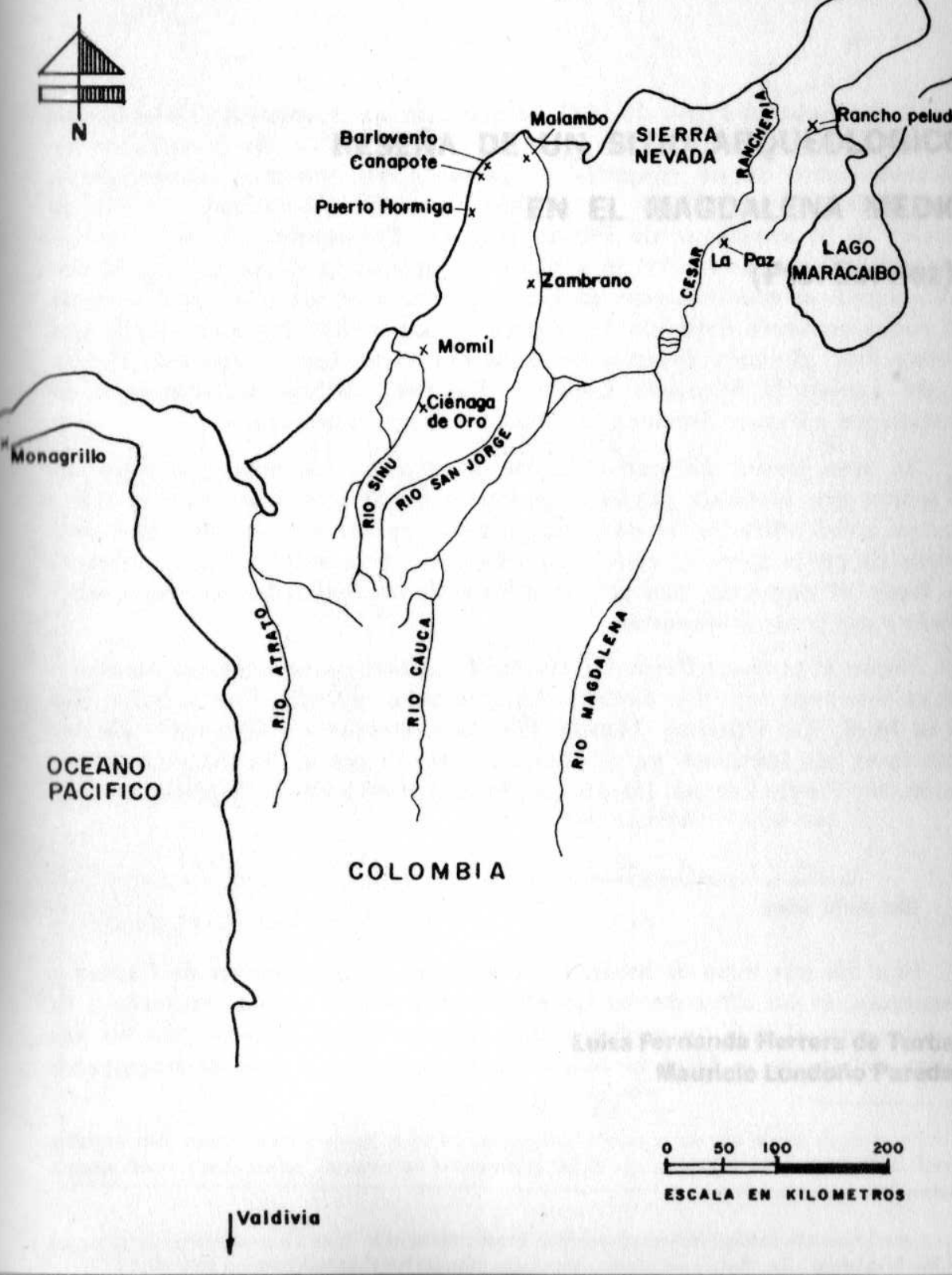